\title{
Optimized Regressor Forest for Image Super-Resolution
}

\section{Chia-Yang Chang}

cychang@media.ee.ntu.edu.tw

Wei-Chih Tu

wctu@media.ee.ntu.edu.tw

Shao-Yi Chien

sychien@ntu.edu.tw
Media IC and System Lab

Graduate Institute of Electronics

Engineering

National Taiwan University

Taipei, Taiwan

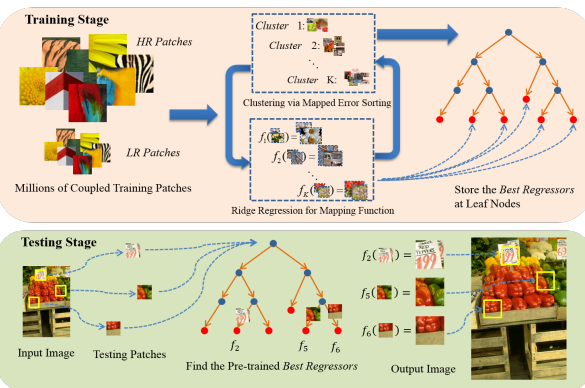

Figure 1: Overview of our framework.

The goal of image super-resolution is to recover missing high frequency details of an image given single or multiple low-resolution images. It is a well-known ill-posed problem and requires mature prior knowledges or enough examples to restore high-quality high-resolution images. Recently, many methods $[1,2]$ formulate image super-resolution as a regression problem. Input image patches are classified into pre-trained clusters, and cluster-dependent mapping functions are employed to super-resolve input patches. The classification following the regression training scheme has a potential problem that the regression step minimizes the error within a cluster. While there might be outliers in the classification process, the learned regression function may not be the optimal for all patches. The key towards high quality and efficient image super-resolution turns to answering the following questions:

(i) How to classify image patches such that patches in the same cluster can be accurately super-resolved using the same regressor?

(ii) How to learn the best regression function such that the super-resolved HR patches have highest numerical accuracy?

In this paper, we tackle the problem in a reverse manner. We put the regressor at the first place. We propose to learn a set of regression functions from training samples using the EM-

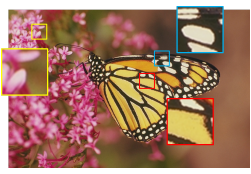

(a) Ground Truth

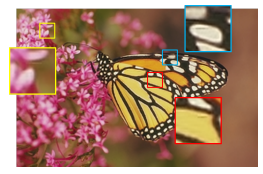

(c) $\mathrm{A}+$

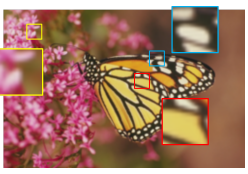

(b) Bicubic

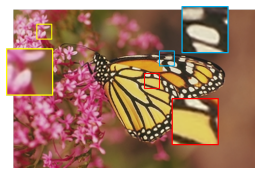

(d) Our ORF-PSNR
Figure 2: Butterfly image from Set14 dataset with upscaling $3 \mathrm{x}$.

algorithm. These regressors are learned to minimize overall reconstruction distortion (e.g. peak signal to noise ratio(PSNR) or structure similarity(SSIM)) for all training data. After that, we will obtain a set of patch-regressor pairs. We then train a random forest from these patchregressor pairs to predict the best regressors for input patches. We call it the Optimized Regressor Forest for super-resolution. An overview of the proposed framework is illustrated in Fig. 1. Our experimental results show that the proposed method is able to achieve comparable or better results in numerical evaluation or visual comparison(see in Fig. 2).

[1] J. Salvador and E. Pérez-Pellitero. Naive Bayes Super-Resolution Forest. In IEEE International Conference on Computer Vision, pages 325-333, 2015.

[2] Radu Timofte, Vincent De Smet, and Luc Van Gool. A+: Adjusted anchored neighborhood regression for fast super-resolution. In Asian Conference on Computer Vision, pages 111-126. 2014. 